\title{
Initial evaluation of Manitoba's cannabis surveillance system
}

\author{
Anja Bilandzic, MPH (1); Songul Bozat-Emre, PhD $(2,3)$
}

Tweet this article

\begin{abstract}
The Government of Manitoba created a cannabis public health surveillance system in 2018 in preparation for nonmedical cannabis legalization on 17 October, 2018. An initial evaluation was conducted to assess the usefulness, flexibility and simplicity attributes of the system, using an online stakeholder survey, website metrics, system analysis and interviews. Resulting recommendations included creating a detailed communication plan for surveillance products, changing the format and frequency of reporting, maintaining strong relationships with partners and building towards a centralized provincial substance use surveillance database and surveillance system.
\end{abstract}

Keywords: cannabis, public health surveillance, evaluation, Manitoba

\section{Introduction}

Nonmedical cannabis use was legalized in Canada on 17 October, 2018, when the Cannabis Act came into force. ${ }^{1}$ To prepare for this event, the provincial department of health (Manitoba Health, Seniors and Active Living) created Manitoba's cannabis surveillance system. The purpose of the system was " $\ldots$ to manage, analyze, and interpret cannabis and related data from a range of stakeholders to provide epidemiologic evidence to inform policy and programs in Manitoba." ${ }^{2}$ More specific objectives of the system were to

(1) monitor cannabis-use behaviour patterns among people in Manitoba;

(2) measure cannabis-related health impacts among people in Manitoba; and

(3) measure justice-related impacts of cannabis policy in Manitoba.

A list of indicators in the system can be seen in Table 1. An initial baseline report was released in November 2018, ${ }^{2}$ and was modelled from existing opioid misuse and overdose surveillance reports. ${ }^{3}$ In order to better understand the performance of the cannabis surveillance system, and to inform future development at this early stage, an initial evaluation of the system was conducted.

The objectives of this initial evaluation were to (1) understand if Manitoba's cannabis surveillance system was meeting its intended objectives, and (2) to define the direction that a sustainable and relevant provincial cannabis surveillance system should take going forward. Specifically, these objectives relate to the content of the system (i.e. which indicators should be used), the identification of stakeholders' needs and the format and frequency of reporting.

\section{Methods}

We undertook an evaluation using guidance from the Centers of Disease Control and Prevention ${ }^{4}$ (CDC) and the European Centre for Disease Prevention and Control, ${ }^{5}$ with specific direction from recent work from the $\mathrm{CDC}$ in evaluating behavioural health surveillance systems. ${ }^{6,7}$ Three attributes, deemed to be the most relevant for a new and changing system, were selected for evaluation: usefulness, flexibility and simplicity. For each attribute, we used multiple evaluation methods (described below), and then made a global assessment based on these results to summarize

\section{Highlights}

- Evaluating public health surveillance systems is essential for understanding system performance and providing guidance for improvement.

- An initial evaluation of Manitoba's cannabis surveillance system showed that the system was moderately useful, highly flexible and moderately simple.

- Recommendations included creating a detailed communications plan prior to surveillance product release to increase the audience reach; producing a shorter infographicstyle product that sets cannabis in context of other substances once or twice a year; and leveraging the existing provincial opioid misuse and overdose surveillance system to include cannabis and other substances.

and communicate our findings. For example, when assessing the overall usefulness (i.e. low, moderate or high), we considered results from an online survey and from website metrics to make a judgment within our organizational context.

\section{Usefulness}

This attribute measures whether the system and its outputs are helpful and important for stakeholders. We assessed usefulness through an online survey and website metrics. We conducted the survey using a snowball approach, whereby key stakeholders who received the survey were instructed to send it to others in their networks. The survey covered topics relating to the overall usefulness of the surveillance system baseline report, specific

\section{Author references:}

1. Canadian Field Epidemiology Program, Public Health Agency of Canada, Winnipeg, Manitoba, Canada

2. Information Management and Analytics Branch, Manitoba Health, Seniors and Active Living, Winnipeg, Manitoba, Canada

3. Max Rady College of Medicine, Rady Faculty of Health Sciences, University of Manitoba, Winnipeg, Manitoba, Canada

Correspondence: Anja Bilandzic, 300 Carlton Street, Winnipeg, MB R3B 3M9; Email: anja.bilandzic@canada.ca 
TABLE 1

Manitoba cannabis surveillance system indicators, by objective

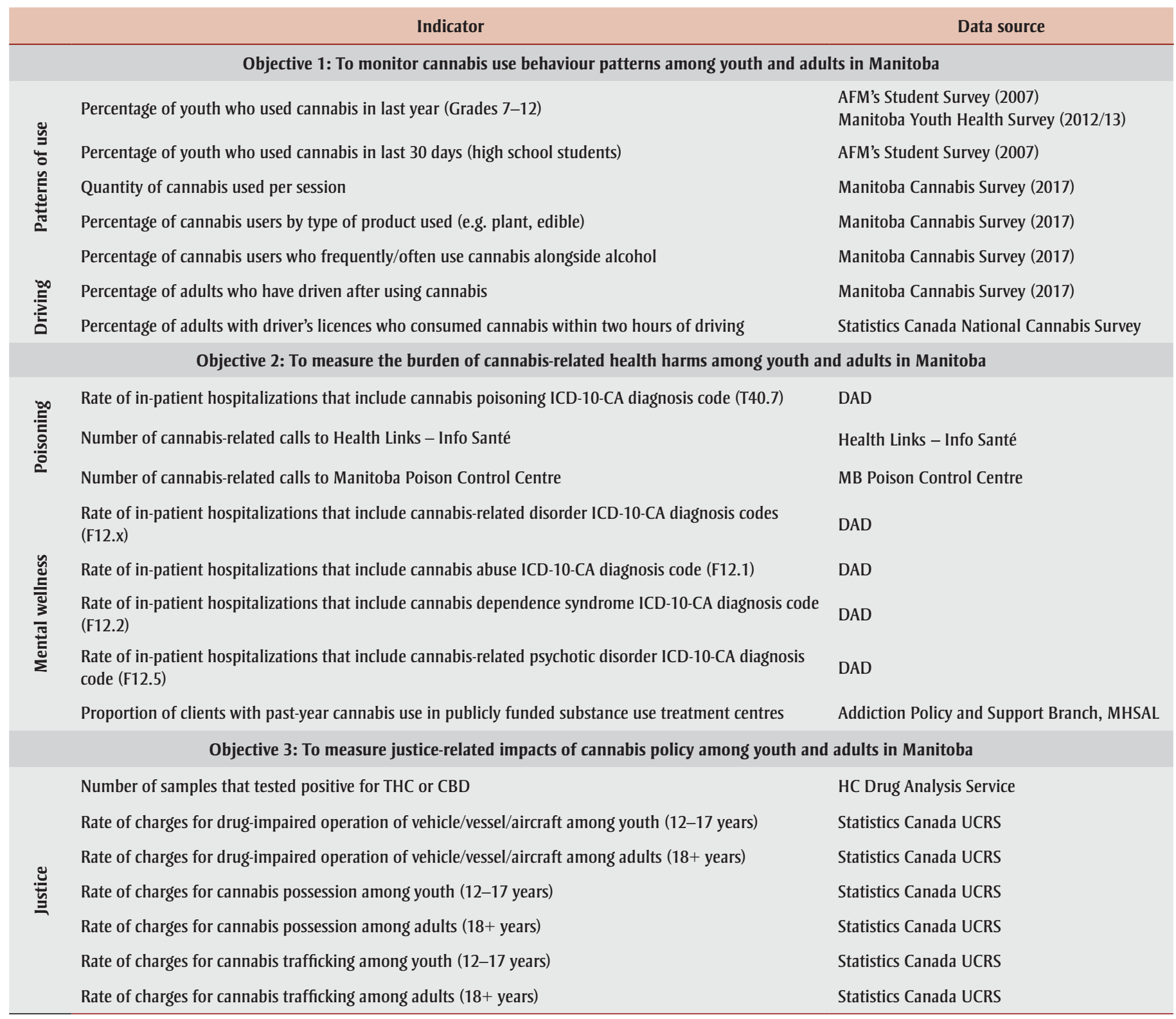

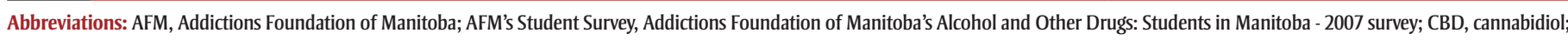
DAD, (hospital) Discharge Abstract Database; HC, Health Canada; MHSAL, Manitoba Health, Seniors and Active Living; THC, tetrahydrocannabinol; UCRS, Uniform Crime Reporting Survey.

indicator and content questions, and direction for the future. (A copy of the survey is available upon request.) The website metrics measured the number of users accessing the report landing page, and their characteristics in the three months following the release of the baseline report.

\section{Flexibility}

This attribute refers to the ability of the system to adapt to changes in stakeholder needs. We assessed flexibility by analyzing the system as a whole (i.e. is it possible to add, delete or modify indicators?).
We also created hypothetical scenarios for adding a new indicator (cannabis poisoning in children) using each of the existing health-related data sources. We consulted external data providers about feasibility and process questions when required.

\section{Simplicity}

This attribute refers to the system structure and how easy it is to use. We assessed simplicity by analyzing the system as a whole (i.e. how many organizations, data types and human resources are needed) and by documenting and analyzing the data collection, management and analysis steps of the surveillance cycle for each data source.

\section{Results}

\section{Usefulness}

Initially, we sent the online survey to 52 stakeholders in Manitoba; after snowball sampling, there were 62 survey respondents. The largest proportion of respondents were from regional public health $(44 \%)$, followed by provincial public health $(21 \%)$, and other provincial departments (16\%). The remainder were 
from other areas of the government or crown corporations, nongovernmental organizations and other agencies.

The key findings of the survey were as follows:

- Most respondents (55\%) were not aware of the baseline report; $24 \%$ were aware but had not reviewed it, and $21 \%$ were aware and had reviewed it.

- Eighty-three percent of respondents said the overall report was somewhat or very useful; $63 \%$ said they already have used or plan to use the information in their work.

- The two most common intentions for using the baseline report were to influence education and awareness activities, and to provide general context to other work.

- Behavioural indicators were most highly rated for usefulness, followed by cannabis-related disorder hospitalizations (ICD-10-CA: F12). In general, poisoning information was rated to be less useful.

- Most respondents (74\%) preferred to see the cannabis data in the context of other substances.

- Most respondents (65\%) preferred a shorter, infographic style of reporting.

- Annual reporting was favoured by $41 \%$ of respondents, and semi-annual by $31 \%$.

Available website metrics captured unique page views for the cannabis surveillance website. Metrics on the report access itself (PDF) were not available. Key findings of the website metrics assessment for usefulness were as follows:

- There were 191 page visits; 56\% were internal government views, and $44 \%$ were external views.

- Almost all internal views were from a desktop computer; for external views, $55 \%$ were from a desktop computer, $38 \%$ from a mobile device and $7 \%$ from a tablet.

- Forty-five percent of all views were directed from a Google search, 26\% were from a direct link and $7 \%$ were from the government search engine;
$18 \%$ of external views came from a Facebook link.

\section{Flexibility}

Overall, the whole system is highly flexible. This is because the indicator groups operate independently, so changing one will have no effect on others (i.e. one indicator is not dependent on the presence of another). Also, indicator change decisions are informal and made through a limited group of stakeholders, which makes the process timely and flexible. However, this structure may pose threats, such as inconsistencies in data over time due to changes, in the absence of good documentation.

When hypothetically adding a new indicator about cannabis poisoning among children from existing poisoning data sources, flexibility differed depending on the data source. For example, for hospitalizations, because an ICD-10-CA code already exists for cannabis poisoning and there is direct access to the data source (including custom age queries), it is relatively simple to get this information. However, other queries outside of the current ICD-10-CA structure would require much more effort and time to modify coding. For other data sources, high level indicators were available but there was low flexibility for more specific information; challenges in current data structures, resources and data sharing agreements were identified. A common theme was that several data quality improvement initiatives were currently underway or planned in the near future, highlighting the importance of continued relationship building with data providers.

\section{Simplicity}

The overall system itself is complex due to the many data sources (ten data sources provided by eight organizations), and types of data included (survey data, administrative data and program data; Figure 1). In terms of resources, the system development and reporting took one full-time employee (FTE) approximately four months to complete, with additional support from a senior epidemiologist (about 0.33 FTE), and publication support for an additional 2.5 months.

For each data source, data collection ranges from downloading publicly available content, to making specific data requests, to accessing departmental data directly. Several file types are involved, including PDFs, Excel and CSV files and SAS code/extracts. In terms of management, these data are stored in different folders on a shared drive. As the number of reports increases, there is a risk that the volume of data will become difficult to manage and document in the current structure. However, the actual analysis of the data is simple; most data sources are already aggregated and analyzed, and a few only require manipulation for data visualization. One data source has an automated process (hospital discharge abstract database).

\section{Recommendations}

- Create a detailed communications plan prior to surveillance product release; consider new media accessing the products (e.g. mobile devices) and platforms (e.g. Facebook).

- Release surveillance products once or twice per year; include a shorter, infographic-style product, and set cannabis surveillance in the context of other substances.

- Review stakeholder indicator survey feedback and explore ways to measure suggested concepts.

- Document methodologies used by each data provider to maintain comparability across surveillance products in future.

- Maintain strong relationships with data providers in order to leverage future opportunities for improvements in data quality.

- Leverage opioid data management and analysis structure to standardize data management, reduce filing inconsistencies and increase automation.

\section{Conclusion}

Overall, this initial evaluation of Manitoba's cannabis surveillance system identified strengths, weaknesses and opportunities for its enhancement. Stakeholders identified useful indicators from all three objectives of the system, and provided feedback for content areas of interest. The system was found to be generally flexible, and to be relatively complex in terms of data volume and management. Key challenges were identified as the limited reach of the baseline report, and the risk of data management inconsistencies for the future. 
FIGURE 1

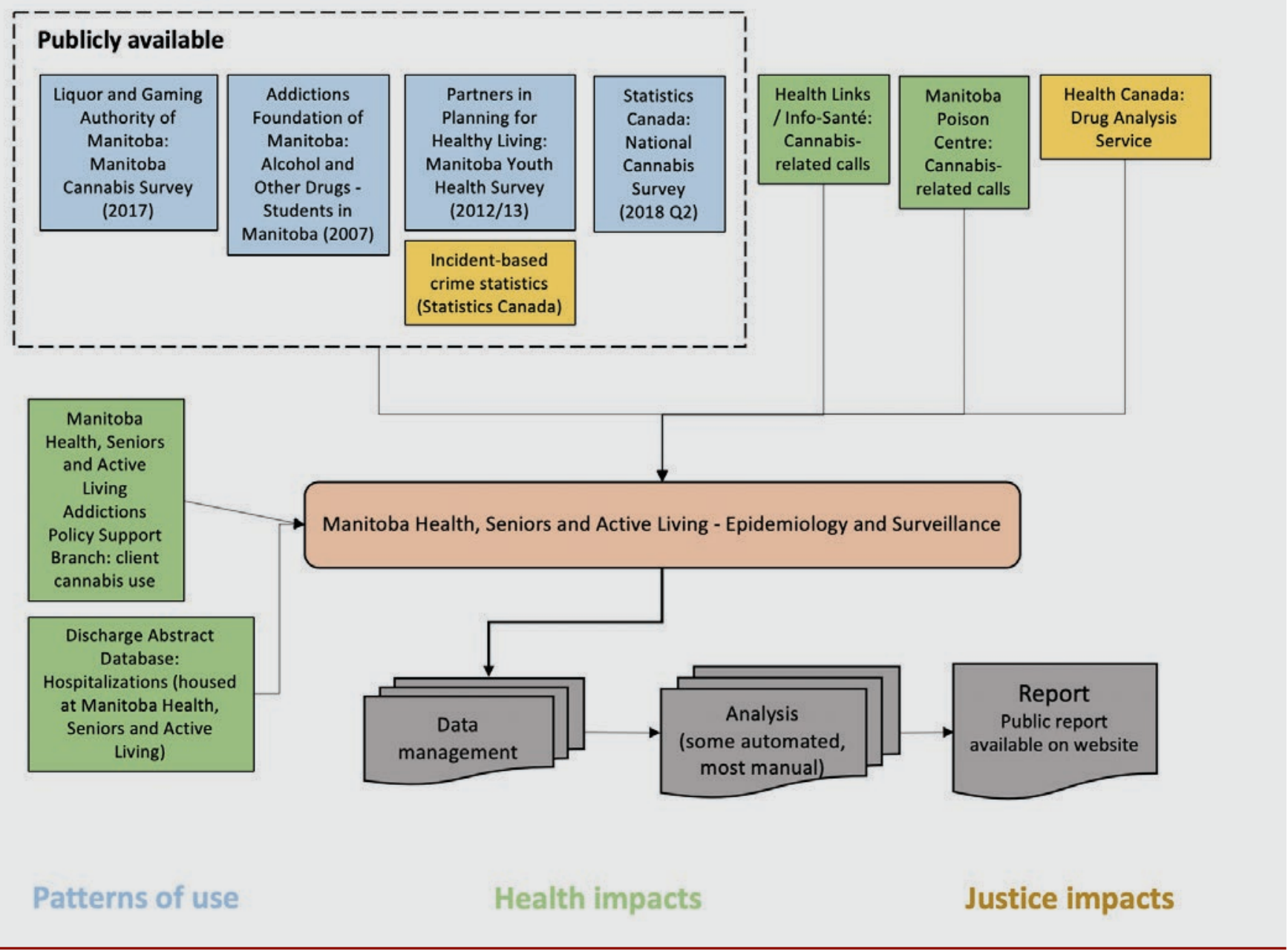

Our experience shows the importance of regular surveillance system evaluation. During the surveillance cycle, this is a step that is often overlooked but really should be a key consideration during design and planning of a surveillance system. It is important that the effort put into maintaining surveillance systems and the processes of sharing information be efficient and serve the information needs of users. Evaluation allows us to understand these needs better and informs how we can adapt and improve processes and surveillance products going forward.

\section{Acknowledgements}

Thank you to Laura McDougall, Carla Loeppky, François-William Tremblay and Philippe Belanger, who provided thoughtful insight and revisions during the evaluation design process. Thank you to Ciara Shattuck for content expertise, and to
Michael Paille for work in the design of the Manitoba cannabis surveillance system.

\section{Conflicts of interest}

The authors have no conflicts of interest to declare.

\section{Authors' contributions and statement}

AB conceptualized and designed the evaluation, analyzed and interpreted the data and drafted the manuscript. SBE reviewed the evaluation and contributed to the drafting of the manuscript.

The content and views expressed in this article are those of the authors and do not necessarily reflect those of the Government of Manitoba or of the Government of Canada.

\section{References}

1. Cannabis Act (S.C. 2018, c. 16) [Internet]. Ottawa (ON): Government of Canada; 2018 [modified 2019 Oct 17; cited 2018 Nov 1]. Available from: https://laws -lois.justice.gc.ca/eng/acts/c-24.5/

2. Manitoba Health, Seniors and Active Living. Surveillance of cannabis in Manitoba [Internet]. Winnipeg (MB): Government of Manitoba; 2019 [cited 2019 Feb 12]. Available from: https:// www.gov.mb.ca/health/publichealth /surveillance/cannabis.html

3. Manitoba Health, Seniors and Active Living. Surveillance of opioid misuse and overdose in Manitoba [Internet]; 2019 [cited 2019 Feb 12]. Available from: https://www.gov.mb.ca/health /publichealth/surveillance/opioid.html 
4. Centers for Disease Control and Prevention. Updated guidelines for evaluating public health surveillance systems. MMWR Recomm Rep. 2001; 50(RR13):1-35.

5. European Centre for Disease Prevention and Control (ECDC). Data quality monitoring and surveillance system evaluation: a handbook of methods and applications. Stockholm (Sweden): ECDE; 2014. 100 p.

6. Azofeifa AS, DF; Lyerla, R; Largo, T; et al. Evaluating behavioral health surveillance systems. Prev Chron Dis [Internet]. 2018 [cited 2019 Jan 21]; 15:170459. doi:10.5888/pcd15.170459.

7. Hagemeyer A, Azofeifa A, Stroup DF, Tomedi LE. Evaluating surveillance for excessive alcohol use in Mexico. Prev Chron Dis [Internet]. 2018 [cited 2019 Jan 21];15:180358. doi:10.5888 /pcd15.180358. 